Jörg Frey and Craig R. Koester (eds.), Signs und Discourses in John 5 and 6. Historical, Literary and Theological Readings from the Colloquium Ioanneum 2019 in Eisenach, WUNT 463, Mohr Siebeck, Tübingen 2021, 386 S., ISBN: 978-3-1610006-7

\title{
Hans Klein
}

In der hier publizierten Aufsatzsammlung sind die für den Druck bereitgestellten Referate veröffentlicht, die anlässlich des 4. Colloquiums Johanneum in Eisenach im August 2019 gehalten wurden. Untersucht werden die Kapitel 5-6 des 4. Evangeliums. Diese beginnen beide mit einer bzw. zwei Wundererzählungen, woran sich eine lange Rede anschließt. Thematisiert werden in den Aufsätzen Christologie, Soteriologie und Eschatologie. In der gegenwärtigen Forschung wird die Frage eifrig diskutiert, ob gegenwärtige und futurische Eschatologie zusammen auf den Evangelisten zurückgehen oder ob die futurische Eschatologie nachträglich eingebracht wurde. Ebenso kontrovers ist auch, ob die Worte Jesu, wonach er das Brot des Lebens ist, das geistlich im Wort und kultisch im Abendmahl vermittelt wird, auf denselben Autor zurückgehen. Da Joh 21 ein Nachtragskapitel ist, ist die These, dass das Johannesevangelium bei der Herausgabe auch ergänzt wurde, naheliegend. $\mathrm{Da}$ es aber in Joh 6 keine parallelen Aussagen zwischen Joh 21 und den als Nachträgen postulierten Texten in Joh 5-6 gibt, kann die Frage, inwieweit man mit einer späteren Redaktion des Evangeliums rechnen muss, nur an den betreffenden Texten entschieden werden. Dafür bedarf es Argumente, die verschieden ausfallen können. Beide Meinungen werden von einigen Autoren vertreten. Eine Reihe der hier zu Wort Kommenden stellt sich diese Frage nicht, sie konzentrieren sich auf die Aussagen in den beiden Kapiteln, wobei sie gerne auch die aktiven Personen genauer ins Blickfeld nehmen.

George Parsenius stellt in „The «Man in the Pool» and the «Man Born Blind»: Comparison in the Lives of Plutarch and the Gospel auf John" (S. 1-13) fest, dass die Erzählungen von der Heilung des Mannes am Teiche Bethesda in Joh 5 und von der des Blindgeborenen in Joh 9 viele Parallelen aufweisen. Diese Beobachtung führt ihn zur These, dass der 4. Evangelist bewusst Menschenpaare nebeneinanderstellt, wie es Plutarch in seinen Parallelbiographien getan hat, wo er einen griechischen und einen römischen „Helden“ miteinander vergleicht. So wie es Plutarch vor allem darum ging, die Charaktere der von ihm beschriebenen „Helden“ herauszustellen und damit zum Nachahmen derselben anzuhalten, so tue das auch Johannes, wenn er

\footnotetext{
* Hans Klein, Prof. em. Dr. Theol., Evangelische Fakultät der Universität Lucian Blaga Sibiu; Str. Negoi, Nr. 47, Sibiu; hansheideklein@gmail.com.
}

RES 13 (3/2021), p. 523-528

DOI: $10.2478 /$ ress-2021-0048 
etwa Maria und Marta oder Thomas und den Lieblingsjünger unterschiedlich zeichnet. Gegensätzlich werden die Jünger einerseits und die Juden andererseits beschrieben. Aber der Mann am Teich Bethesda wie auch Nikodemus bleiben in der Schwebe (ambiguous charakter). Ihr Verhalten soll die Leser anhalten, selbst über ihr eigenes Leben in seiner Beziehung zu Jesus nachzudenken.

Christos Karakolis folgt in „The Lame Man (John 5,1-18) as a Model for the Johannine Jews. A Narrative and Reader Response Analysis“ (S. 15-28) in gewisser Weise diesem Modell. Er sieht in dem Mann am Teiche Bethesda einen Judenchristen beschrieben, der Hilfe von Jesus braucht und auf Jesu Anweisungen hin gehorsam reagiert und dafür Gott im Tempel dankt, der aber auch gerufen ist, bei Jesus zu bleiben, während die Führerschaft der Juden Jesus ablehnt. Die Leser des Evangeliums sind zum Überdenken ihrer Situation gerufen.

Adele Reinhartz geht in „Doing God's Word: John 5,17-18 from a Jewish Perspective" (S. 29-38) den Fragen nach, ob Gott am Sabbat arbeitet, wie in Joh 5,17f ausgesprochen ist, und was es bedeutet, dass Jesus wie Gott wirkt. Wiewohl in Gen 2,3 davon gesprochen wird, dass Gott von seinen Werken ruhte, setzt das Judentum voraus, dass er weiterhin in der Natur und als Richter wirkt. Hingegen soll der Mensch nicht arbeiten. Wenn Jesus seine Heiltätigkeit von Gottes Wirken auch am Sabbat ableitet, setzt er sich nicht Gott gleich, wie ihm die Gegner vorwerfen, die beiden sind allerdings in ihrem Wirken gleich.

Von zentraler Bedeutung ist der Beitrag von Jörg Frey: Who Should „Not Wonder"? On the Audience and Logical Structure of the DiscourseSection John 5,19-30 (S. 39-58), in dem er für die Einheit und wohlüberlegte Konstruktion des Abschnittes plädiert. Er hält zunächst fest, dass in 5,17f Gott in Jesu Heilungswunder neu zu wirken beginnt und geht sorgfältig der Struktur sowie den semantischen und linguistischen Eigenheiten des Abschnittes nach, wobei er einen Unterschied zwischen dem Gebrauch des Wortes „wundern“ in V. 20 und V. 28 festhält. Es liegt ihm daran, die Einheitlichkeit des Abschnittes herauszustellen. Da seit J. Wellhausen über R. Bultmann und bis heute (S. 48f) der Abschnitt 5,28f-der im Unterschied zu den dominierenden Aussagen des Evangeliums über die Notwendigkeit einer jetzigen Entscheidung zu Jesu Worten, eine futurische Eschatologie enthält - als spätere Hinzufügung angesehen wird, wirft er diesen Forschern vor, ihr Blick sei durch systematisch-theologische Voraussetzungen verdunkelt worden (have blinded the eyes, S. 57), sie hätten den Charakter des Abschnittes verkannt (als ob eine systematisch-theologische Prägung nicht auch den Blick für gewisse Einsichten schärfen könnte). Die Argumente, die J. Frey anführt, hängen an der Einschätzung der unterschiedlichen Aussagen, die man ein- 
heitlich oder differenziert sehen kann. Der Beitrag von M. Theobald, der sich diesem Problem ebenso stellt, kommt zu einem anderen Schluss (siehe unten). Schwierig wird die Tatsache erst, wenn mit der Beobachtung des Unterschiedes eine Wertung vorgenommen wird. Die Verse 5,28f gehören zum Evangelium, unabhängig davon, ob sie der Grundschrift oder dem Nachtrag zugeschrieben werden. Die Differenzierung aber ermöglicht, die Zielrichtung des Evangeliums und jene der späteren Nachträge schärfer zu sehen.

Eine ähnliche Sicht wie J. Frey vertritt R. Alan Culpepper in „Jesus the Judge (John 5,21-30). The Theme of Judgment in John's Gospel“ (S. 59-86), der wie Frey präsentische und futurische Eschatologie demselben Autor zuschreiben kann. Für seine Sicht zieht er die Aussagen in der Zeit des zweiten Tempels und jene der Synoptiker heran und wirft dann einen Blick auf die gesamten johanneischen Schriften. Er zeigt, dass der 1. Johannesbrief eine eindeutig futurische Eschatologie vertritt und diese auch in den (doch wohl redaktionell eingefügten) stereotypen Formeln „Ich werde ihn erwecken am jüngsten Tag" in 6,39.40.44.54 begegnen. In seiner Sicht übernimmt Johannes traditionelle Aussagen vom Endgericht und zeichnet sie in sein christologisch-soteriologisches Konzept ein.

Ruben Zimmermann und Zacharias Shoukry widmen sich ausführlich dem Thema „Creatio Continua in the Fourth Gospel. Motifs of Creation in John 5-6" (S. 87-115). Sie gehen darin den unterschiedlichen Aussagen über die Schöpfung, aber auch Gottes und Jesu Wirken in der Schöpfung und dem Verhalten der Jünger mit Teilen der Schöpfung nach und zeigen damit eine neue Möglichkeit der Lektüre des Johannesevangeliums aus der gegenwärtigen Sicht der bedrohten Schöpfung auf.

In seinem Beitrag „The Construction of Space in John 5-6“ (S. 117-27) betont Jean Zumstein, dass die neutestamentlichen Forscher im 19. Jh. eifrig die zeitlichen Angaben im NT untersucht haben, die räumlichen wären etwas vernachlässigt worden. Er möchte sich den Aussagen über den Raum im Johannesevangelium widmen, die gegenüber jenen über die Zeit eine erhöhte Bedeutung haben, und untersucht dazu Kap 5-6. Er findet dort erkennbare und nicht identifizierbare Ortsangaben, aber auch, dass über Jesu Bewegung an verschiedene Orte berichtet wird. Räumlich sind auch die Angaben Jesu im Verhältnis zu seinem himmlischen Vater. Er ist herabgestiegen und fährt wieder hinauf.

Michael Labahn vergleicht in seinem Beitrag „Jesus: Gottes Hilfe in der Not. Theologische und christologische Charakterisierung Jesu und seiner Jünger im Vergleich zwischen Mk 6,30-52 und Joh 6,1-21" (S. 129-54) die beiden Abschnitte bei $\mathrm{Mk}$ und Joh hinsichtlich der Speisung der 5.000 und dem anschließenden Seewandel Jesu. Dabei betont er, dass 
die Berichte der beiden Evangelisten sehr ähnlich sind und vergleicht das Verhalten Jesu als des Gebers in der Not und das der Jünger in den beiden Varianten. Bei Markus ist Jesus als Hirte gekennzeichnet, der die Seinen mit Nahrung versorgt, bei Johannes wird er von der Menge als König verstanden. Beide Verständnisse weisen auf das Kreuz hin. Bei Markus wird das Jüngerunverständnis im Zusammenhang der Speisung betont, bei Johannes sind die Jünger als Diener Jesu gekennzeichnet. Die kleinen Unterschiede bei Markus und Johannes entsprechen ihrer unterschiedlichen Theologie.

Craig R. Koester untersucht in seinem Beitrag „Signs and Christology in John 6,1-21 in Light of Jewish and Greco-Roman Frames of Reference. Prophet, King and Revealer of God" (S. 155-73) die Christologie des Johannes ausgehend von der Speisungs- und der Seewandelgeschichte. Er findet, dass Jesus zu Recht als Prophet bezeichnet wird, wobei sowohl an den Propheten Mose (Dt 18) als auch an Elia (Joh 1,25) gedacht ist, weil er Gottes Wort, wie Mose, und Wunder, wie Elia, nahebringt. Auch die Bezeichnung als König treffe zu, auch wenn er nicht als König dieser Welt verstanden werden will. Er bringt den Menschen in der Speisung Brot, wie es die Könige versprachen, was in römische Münzen im Bilde vom Füllhorn eingeprägt ist. In der Formel „Ich bin es“, angesichts der ängstlichen Jünger im Sturm des Meeres, nimmt Jesus die Gottesbezeichnung „Ich bin“ auf und schafft Ruhe im Sturm, wie es von dem Gott Israels erwartet wird.

Catrin H. William, „Intertextual Perspective on John's Sea-Crossing Account (John 6,16-21)“ (S. 175-97), geht in der Frage, inwieweit die Erzählung des Johannes vom Seewandel Jesus von jener des Markus abhängig sei, von der These aus, dass Johannes den markinischen Bericht wie einen intertextuellen Text behandele, ihn also in derselben Weise verwende, wie er die alttestamentlichen Zitate heranzieht. So habe die Erscheinung Jesu vor den Jüngern Motive der Theophanie übernommen. Die Selbstbezeichnung Jesu mit „Ich bin (es)“ führe zur folgenden Aussage „Ich bin das Brot des Lebens“. Diese Selbstbezeichnung sei aber nicht von der MannaWundergeschichte her zu verstehen, im Hintergrund derselben stünden die Aussagen über die Selbstbezeichnung Gottes bei Deuterojesaja. Dazu gehöre auch die bei Deuterojesaja oft gebrauchte Formel „Fürchte dich nicht“, die Jesus den Jüngern mit seinem „Fürchtet euch nicht“ zuspricht. Wenn die Menge aufgrund der Speisung Jesus als Propheten wie Mose ansieht, ist sie im Irrtum. Nicht der erste Exodus, sondern der von Deuterojesaja verkündete neue Exodus präge die johanneische Seewandelgeschichte.

Weitgehend systematisch-theologisch orientiert ist der Beitrag von William Loader „Soteriology and Spirituality in John 6. A Relecture on Key Issues in Johannine Theology" (S. 199-213). Er hebt in dessen erstem Teil 
die Heilsbedeutung des Todes Jesu hervor, an der Jesus Anteil gibt, wie Joh 6,27 und 6,51-28 hervorheben. Hier und in soteriologischen Aussagen wie der, dass Jesus der Welt Sünde trägt (Joh 1,29) oder dass er sein Leben für ... einsetzt (Joh 10,11.15: 15,13), nimmt Johannes die Sprache der Tradition auf. Für diesen Evangelisten ist aber ebenso wichtig, dass Jesus als der Irdische das Licht der Welt, das Brot des Lebens ist und nicht nur gibt, er also auch in seiner irdischen Seinsweise zum Heil der Menschen, der Welt wirkt, Gott nahebringt. Beides gehört zusammen.

Michael Theobald, „Diese Rede ist hart“ (Joh 6,63). Wider die Versuchung Joh 6,51c-58 bildlich zu verstehen (S. 215-48), macht glaubhaft, dass die Aussage „diese Rede ist hart“ sich auf die Brotrede 6,36-51d rückbezieht, die den Herabgestiegenen und im Kreuz erhöhten Menschensohn thematisiert. V.51c-58 ist als nachträgliche Erläuterung anzusehen, welche die Fleischwerdung des Menschensohnes hervorhebt und an den variierten Abendmahlsworten festmacht: Der irdische Jesus muss als solcher ernst genommen werden. Von da aus sind auch andere spätere Erläuterungen innerhalb des Johannesevangeliums zu beurteilen.

Udo Schnelle, „Johannes 6,60-71 und die Genese der johanneischen Theologie“ (S. 249-73), findet in Joh 6 wichtige „Schlüsselbegriffe“ johanneischer Theologie und geht davon aus, dass der Gedanke der Leiblichkeit Jesu von Johannes in besonderer Weise gegenüber Doketisten hervorgehoben wird. Damit erneuert und spezialisiert er seine bekannte, von G. Strecker übernommene These, dass das Johannesevangelium nach den Briefen des Johannes geschrieben wurde. In beiden Schriften wird die Leiblichkeit Jesu hervorgehoben, wird das „Weggehen“ einiger Glaubenden/Jünger thematisiert. Dass die Speisung samt Seewandel und Petrusbekenntnis für die Theologie des Johannes von zentraler Bedeutung sind, lasse sich schwerlich bestreiten. Vielleicht habe der Evangelist sogar eine Kombination dieser drei Berichte als Tradition vorgefunden, die er ausgestaltete. Im Hinblick auf die Reihenfolge der johanneischen Schriften scheint es mir plausibler, wenn man das Johannesevangelium als älter ansieht als den 1. Johannesbrief. Denn nach 8,31-47 gehen Glaubende (Judenchristen) ins Judentum zurück. Ihnen gegenüber wird die geistliche Höhe hervorgehoben. Nach dem 1. Johannesbrief aber glauben die Gegner nicht an die Fleischwerdung, die Spiritualität ist ihnen nicht hoch genug.

Marianne Meye Thomson, „The Offense of Jesus's Death in the Gospel of John (John 6,60-71)“ (S. 275-88), beschreibt in ihrer Studie die Reaktionen der zunächst glaubenden Menge, die ihn aufgrund der Speisung zum König machen will, aus der aber viele ihn nach seiner Brotrede und seinen Worten über sein Fleisch und Blut verlassen. Einen besonderen Fall 
stellt Judas dar, der ihn zwar zunächst nicht verlässt, aber am Ende verrät. Er wird als „Teufel“ identifiziert. Die Jünger haben auch selbst ihre Zweifel, beginnend mit Nathanael bis hin zu Thomas. Auch Petrus versagt. Aber er glaubt am leeren Grab. Johannes beschreibt die Zweifel der Jünger und das Weggehen der Vielen, um die Glaubenden zu warnen. Rechtes Leben im Glauben geschieht in der Gemeinschaft mit dem gekreuzigten und auferstandenen Herrn.

D. Francois Tolmie, „Elected and a Devil. The Characterization of Judas Iscariot in the Fourth Gospel“ (S. 289-309), geht der Frage nach, wie Judas im Johannesevangelium gezeichnet wird, und klammert die Frage nach dem historischen Judas und nach der Wirkungsgeschichte der neutestamentlichen Texte über ihn aus. Im Durchgang aller Texte, die im Evangelium über Judas handeln, ergibt sich für Tolmie, dass Judas von Jesus erwählt ist, er aber Jesus nicht von Gott gegeben wurde. Er wird immer als negative Person gezeichnet, in 12,4-6 so, dass er als Träger des Beutels geldgierig ist und die Salbung der Maria hinterfragt. Aber Jesus gegenüber ist er nicht aktiv, er sieht zu, wie Jesus gefangen genommen wird. Er gilt als „ein Teufel" und geht in die Nacht, aber ohne für Jesus direkt etwas zu tun, er sieht der Gefangennahme Jesu nur zu. Vielleicht wirft, so Tolmie, Johannes einen Seitenblick auf solche "Jünger" Jesu, die eine abwartende, zweifelnde Haltung gegenüber Jesus, d. h. seiner Gemeinde, haben.

Die Sammlung der Aufsätze ist einem schönen Blumenstrauß mit sehr unterschiedlichen Blumen vergleichbar. Enthalten sind darin sich gegenseitig ergänzende aber auch einander widersprechende Studien, die die Forschung zu weiterem Nachdenken anhalten werden. 